PROCEEDINGS OF THE

AMERICAN MATHEMATICAL SOCIETY

Volume 129, Number 8, Pages 2489-2496

S 0002-9939(00)05904-9

Article electronically published on December 28, 2000

\title{
SEIBERG-WITTEN INVARIANTS FOR MANIFOLDS DIFFEOMORPHIC OUTSIDE A CIRCLE
}

\author{
STEFANO VIDUSSI
}

(Communicated by Ronald A. Fintushel)

\begin{abstract}
In this paper we prove that simple type four manifolds with $b_{2}^{+}>1$ which are diffeomorphic outside a point or outside a wedge of circles have the same Seiberg-Witten invariants, excluding the use of these invariants to detect eventual inequivalent smooth structures.
\end{abstract}

\section{INTRODUCTION}

Manifolds which are diffeomorphic outside a point or outside a wedge of circles are not, in general, diffeomorphic. When we deal with four dimensional manifolds, these manifolds turn out to be (at least) homotopy equivalent, and it would be interesting to know whether some smooth invariants, like Donaldson polynomials or Seiberg-Witten invariants, could detect nondiffeomorphic structures. The aim of this paper is to prove that smooth four manifolds of simple type, with $b_{2}^{+}>1$, diffeomorphic on the complement of a point or a wedge of circles have the same Seiberg-Witten invariants for any $\operatorname{spin}^{c}$ structure, showing that these invariants cannot be used to distinguish the manifolds.

The first of these two cases has been analysed, in the framework of Yang-Mills theory, in [1], where it was proven that the Donaldson polynomials of two such manifolds coincide. For what concerns the second case, we are not aware of any reference, but is seems highly reasonable that, using the gluing theory established in [7] (in the particular the cases discussed in 4]) the same result should be achieved.

As noted in 11, in the case of manifolds diffeomorphic in the complement of a point, two approaches are viable within the framework of Yang-Mills theory: the first is a direct study of the relations of the two Yang-Mills moduli spaces, the second is the observation that two such manifolds admit a quasiconformal homeomorphism and have therefore coinciding Donaldson polynomials, as comes from the work of 3]. For Seiberg-Witten theory the latter approach does not seem available, due to the difficulties that arise in proving a result in the spirit of [3] in the framework of Seiberg-Witten theory. We will therefore use the first approach. In the same way we can also treat the problem of manifolds diffeomorphic in the complement of a wedge of circles.

Received by the editors August 27, 1999 and, in revised form, December 10, 1999.

2000 Mathematics Subject Classification. Primary 57R57; Secondary 57Mxx.

Key words and phrases. Seiberg-Witten invariants, smooth topology of four manifolds.

The author would like to thank Stefano Demichelis for several discussions.

(C)2000 American Mathematical Society 
The main ingredient of our approach is the relation between the SW moduli space of a manifold $M=M^{+} \cup_{N} M^{-}$, decomposed along a three manifold $N$ which has nondegenerate solutions for the three dimensional SW equations, and the moduli spaces of finite energy solutions on the cylindrical end manifolds $\hat{M}^{ \pm}=$ $M^{ \pm} \cup_{N} N \times[0, \infty)$. In our case $N$ will be either $S^{3}$ or $\#_{k} S^{1} \times S^{2}$. As we want to work with moduli spaces, on $M$, which are generically cobordant when we stretch the neck of $M$ along $N$, we will assume that $b_{2}^{+}(M)>1$ (to deal with the case of $b_{2}^{+}(M)=1$ we would need to pay some attention to the identification of the chamber, when relating moduli spaces, but for the sake of simplicity we will omit this case).

We start with a short digression on finite energy moduli spaces on cylindrical end manifolds, referring, e.g., to 10 for a detailed proof.

Let $\hat{X}=X \cup_{N} N \times[0, \infty)$ be a cylindrical end four manifold constructed by gluing an infinite cylinder to a compact manifold $X$ having boundary $N$ of positive scalar curvature. Let $P_{\hat{X}}$ be a $\operatorname{spin}^{c}$ structure on $\hat{X}$ and denote by $P_{N}$ the spin ${ }^{c}$ structure induced on $N$. As is well known (see [6]), once the connection is put in temporal gauge, SW equations on the cylinder assume the form of gradient flow equations for a functional (Chern-Simons-Dirac). We can therefore define the energy of a solution on the cylinder, and this coincides with the variation of the CSD functional over the cylinder. We will denote by $\mathcal{M}\left(P_{\hat{X}}\right)$ the moduli space of solutions on $\hat{X}$ that have finite energy on the cylinder, modulo the action of the gauge group $\mathcal{G}\left(P_{\hat{X}}\right)$. The finite energy condition is the correct one to obtain the desired regularity properties for the moduli spaces on a cylindrical end manifold; see, e.g., 8], Sect. 8.

As $N$ admits a metric of positive scalar curvature it is possible, via Weitzenböck formula, to have a complete knowledge of three dimensional SW theory. In particular the only $\operatorname{spin}^{c}$ structures $P_{N}$ on $N$ which have solutions are those whose determinant bundles $\mathcal{L}_{N}$ are torsion, and these solutions are flat couples $(B, 0)$. The following proposition holds true:

Proposition 1.1. Let $\hat{X}=X \cup_{N} N \times[0, \infty)$ be a cylindrical end four manifold, with $N$ of positive scalar curvature, having $b_{2}^{+}(X)>0$. Up to gauge transformation any finite energy solution of $S W$ equations for a spin ${ }^{c}$ structure $P_{\hat{X}}$ decays with exponential rate to (the pull back of) a reducible solution on $N$, defining a limit map

$$
\partial_{\infty}: \mathcal{M}\left(P_{\hat{X}}\right) \longrightarrow \hat{\chi}(N)
$$

which associates to any gauge equivalence class of solutions $[(A, \psi)]$ its limit $[(B, 0)]$, in the space of reducible solutions on $P_{N}$ modulo those automorphisms of $P_{N}$ which extend to $P_{\hat{X}} ; \mathcal{M}\left(P_{\hat{X}}\right)$ is a compact, smooth (after generic compactly supported perturbation of $S W$ equations), oriented manifold of dimension

$$
\begin{aligned}
& \operatorname{dim} \mathcal{M}\left(P_{\hat{X}}\right)=2 i n d_{\mathbb{C}} \not_{A}-1+b^{1}(X)-b_{+}^{2}(X) \\
& =\frac{1}{4} c_{X}-\frac{1}{4} \sigma(X)-\eta_{B}(0)-1+b^{1}(X)-b_{+}^{2}(X)
\end{aligned}
$$

where

$$
c_{X}(A, \psi):=-\frac{1}{4 \pi^{2}} \int_{\hat{X}} F_{A} \wedge F_{A}
$$


is a well defined locally constant term, which depends only on the spin ${ }^{c}$ structure, and the Dirac $\eta$ invariant is constant on the space of flat connections. The map $\partial_{\infty}$ is smooth and transversal.

A few notes on this Proposition: a) $\hat{\chi}(N)$ is a cover of $\chi(N)$, the space of all $\mathcal{G}\left(P_{N}\right)$-equivalence classes of reducible solutions on $P_{N}$, of fibre $H^{1}(N, \mathbb{Z}) / H^{1}(X, \mathbb{Z})$; b) the compactness property of the moduli space is a consequence of the connectedness of $\chi(N)$, which forbids losses of energy for weak limits on the cylinder; c) there exists an $U(1)$-equivariant version of $\partial_{\infty}$ considering based gauge equivalence classes; d) whenever $N$ has positive $b_{1}, \hat{\chi}(N)$ is nondegenerate but it is obstructed, the obstruction at each point being identified with $H^{2}(N, i \mathbb{R})$ : the Kuranishi model of the solution spaces identifies in fact a neighbourhood of a solution $(B, 0)$ in $\hat{\chi}(N)$ with the zero set of a map

$$
H^{1}(N, i \mathbb{R}) \times k e r \not_{B} \longrightarrow H^{2}(N, i \mathbb{R}) \times \operatorname{coker} \not_{B},
$$

and $\operatorname{ker} \not_{B}\left(=\operatorname{coker} \not_{B}\right)$ vanishes by Weitzenböck formula.

A description of the ungluing process, when we endow $M$ with a metric which stretches a tubular neighbourhood of $N$, shows that a solution decomposes, in the geometric limit of $P_{M}$, in a couple of finite energy solutions on $\hat{M}^{ \pm}$which have compatible limit values w.r.t. the maps $\partial_{\infty}$; the dimension formula described above (ultimately, the presence of the gluing factor due to the reducibility of $\chi(N)$ ) implies that the two solutions on $\hat{M}^{ \pm}$cannot both be irreducible. If we have both $b_{2}^{+}\left(M^{ \pm}\right)>0$ this brings to a vanishing theorem for SW invariants (e.g. in the usual case of connected sum, where $N=S^{3}$ ), while when one of the factors, say $M^{-}$, has $b_{2}^{+}\left(M^{-}\right)=0$, this dictates the rules for the gluing process, which must glue a solution in $\mathcal{M}\left(P_{\hat{M}^{+}}\right)$with a reducible on $P_{\hat{M}^{-}}$. Note that this holds true without any condition on the metric of $M^{-}$, in particular careless whether irreducible solutions on $P_{\hat{M}^{-}}$exist. We denote by $\chi\left(\hat{M}^{-}\right)$the gauge equivalence classes of reducible solutions. This coincides, as manifold, with the gauge equivalence classes of flat connections on $\hat{M}^{-}$but not, in general, as moduli space.

The gluing theory of gauge moduli spaces along smooth points is analysed in 2] and [9] (for its application in SW set up, see [5]). Before we proceed, we need information on the genericity of the moduli spaces we will glue; on one side, the irreducible moduli space $\mathcal{M}\left(P_{\hat{M}^{+}}\right)$, as $b_{2}^{+}\left(M^{+}\right)$will be positive, is generic and unobstructed. For what concerns the reducibles on $P_{\hat{M}^{-}}$, we need a description of the Kuranishi model of a neighbourhood of a reducible solution $(A, 0)$; the linearization of SW equations on the complex of exponentially decaying spinors and forms around a reducible describes this neighbourhood as the zero set of a map

$$
H^{1}\left(M^{-}, N, i \mathbb{R}\right) \times H^{1}(N, i \mathbb{R}) \times \operatorname{ker} \not_{A} \rightarrow H^{1}(N, i \mathbb{R}) / H^{1}\left(M^{-}, i \mathbb{R}\right) \times \operatorname{coker} \not_{A}
$$

which endows $\chi\left(\hat{M}^{-}\right)$with a virtual bundle of real rank $2 i n d_{\mathbb{C}} \not_{A}+b^{1}\left(M^{-}\right)$. Although this looks in general quite a complicated situation, we will have to deal only with cases where it can be simplified, so we will pass to describe the two cases of our interest.

\section{IDENTIFICATION OF THE MODULI SPACES}

As first case we consider two smooth manifolds which are diffeomorphic in the complement of a point. We want to prove 
Proposition 2.1. Let $M_{1}$ and $M_{2}$ be two manifolds with $b_{2}^{+}>1$ diffeomorphic in the complement of a point. Then for any spin ${ }^{c}$ structure the Seiberg-Witten invariants of $M_{1}$ and $M_{2}$ coincide.

Proof. Denote by $\phi$ the diffeomorphism

$$
\phi: M_{1} \backslash p_{1} \longrightarrow M_{2} \backslash p_{2} ;
$$

we can choose contractible neighbourhoods $U_{i}$ of $p_{i}$ in such a way that the image, via $\phi$, of a standard three-sphere $S^{3}=\partial D^{4} \subset D^{4} \subset U_{1}$, where $D^{4}$ contains $p_{1}$, is contained in $U_{2}$. The image $\phi\left(S^{3}\right)$ of the three-sphere separates $M_{2}$ into two connected components, one of which, denoted by $D_{\Gamma}^{4}$, contains $p_{2}$ and is homeomorphic (but not diffeomorphic, in the absence of the generalised Schönfliess conjecture; see 1]) to the four dimensional disc. The two manifolds $M_{1}, M_{2}$ must be homeomorphic but we cannot decide about their diffeomorphism. Denoting $M^{+}=M_{1} \backslash$ int $D^{4}$ we can consider the decomposition of the two manifolds $M_{i}$ given as

$$
M_{1}=M^{+} \cup_{S^{3}} D^{4}, \quad M_{2}=\phi\left(M^{+}\right) \cup_{\phi\left(S^{3}\right)} D_{\Gamma}^{4} ;
$$

the map $\phi$ defines a diffeomorphism $M^{+} \cong \phi\left(M^{+}\right)$and from now on we will identify these two manifolds. The latter observation tells us that SW theory on the two positive factors of the two manifolds is equivalent, and in particular finite energy moduli spaces on the cylindrical end manifolds $\hat{M}^{+}$and $\hat{\phi}\left(M^{+}\right)$coincide. Once we have a $\operatorname{spin}^{c}$ structure $P_{M^{+}}$, which induces the trivial one on $S^{3}$ (this is the only structure which admits finite energy solutions), an obvious cohomology argument shows that it defines a canonical extension $P_{M_{i}}$ on $M_{i}$ constructed by gluing $P_{M^{+}}$ with the product $\operatorname{spin}^{c}$ structure on $D^{4}$ respectively $D_{\Gamma}^{4}$. When we consider the extension of these $\operatorname{spin}^{c}$ structures on the cylindrical manifolds $\hat{D}^{4}$ and $\hat{D}_{\Gamma}^{4}$, the gauge equivalence classes of reducible solutions are identified, as sets, with the trivial connection (as only the homotopy type matters) and the Kuranishi model of a neighbourhood of it appears, according to the description of equation (1.5), as zero set of a map

$$
\operatorname{ker} \not_{A} \longrightarrow \operatorname{coker} \not_{A} \text {. }
$$

The index of this map, i.e. the index of the Dirac operator coupled with the trivial connection, is zero, as follows from Atiyah-Patodi-Singer theorem (suitably adapted to deal with the cylindrical end case; see 7]) because of the fact that all the terms of the index formula vanish. The gluing formula which relates $\mathcal{M}^{o}\left(P_{M_{1}}\right)$ with $\mathcal{M}^{o}\left(P_{\hat{M}^{+}}\right)$is particularly simple: Weitzenböck formula implies the vanishing of $k e r \partial_{A}$ on $\hat{D}^{4}$ and from this we deduce that $\chi\left(\hat{D}^{4}\right)$ is in fact a smooth moduli space cut transversely by SW equations. The absence of obstruction identifies therefore the moduli space $\mathcal{M}^{o}\left(P_{M_{1}}\right)$ with the fibred product

$$
\mathcal{M}^{o}\left(P_{M_{1}}\right)=\mathcal{M}^{o}\left(P_{\hat{M}^{+}}\right) \times_{\chi(N)} \chi\left(\hat{D}^{4}\right)=\mathcal{M}^{o}\left(P_{\hat{M}^{+}}\right) .
$$

The gluing problem for $M_{2}$ is a bit more subtle. In fact we have to notice that we have no information on the metric structure of $\hat{D}_{\Gamma}^{4}$ and so the previous argument cannot be applied directly. In fact, just a little detour is required to obtain the same result as above. The point is that perturbing the equations, as Dirac operator has zero index, we can kill both the kernel and the cokernel associated to the only reducible solution of the perturbed equations: so we perturb the curvature part of the SW equations by adding a term $d^{+} \nu$, where $\nu$ is an exponentially decaying 
term (even compactly supported, if we wish) in $\Omega^{1}\left(\hat{D}_{\Gamma}^{4}, i \mathbb{R}\right)$, i.e. we consider the solutions of the equation

$$
F_{A}^{+}=d^{+} \nu
$$

We can state now a lemma that rules a generic vanishing for the kernel of the Dirac operator coupled to a solution of equation 2.5):

Lemma 2.2. Let $M^{-}$be a manifold which has the homotopy type of $D^{4}$. For a second category subset of perturbations $\eta=d^{+} \nu, \nu \in \Omega_{\delta}^{1}\left(\hat{M}^{-}, i \mathbb{R}\right)$, the kernel of the Dirac operator coupled to a solution of $d^{+} \nu$-perturbed curvature equation vanishes.

Proof. Note that, up to gauge equivalence, the reducible solution of $d^{+} \nu$-SW equations is given by $\left(A_{0}+\nu, 0\right)$ where $A_{0}$ is the trivial connection. We have to check that, for a generic choice of $\nu, \operatorname{ker} \not_{A_{0}+\nu}$ vanishes. In order to do so we consider the Dirac equation as defining a section of the $\Gamma_{\delta}\left(S^{-}\left(P_{\hat{M}^{-}}\right)\right)$-bundle over $\left\{A_{0}\right\} \times \Gamma_{\delta}^{*}\left(S^{+}\left(P_{\hat{M}^{-}}\right)\right) \times \Omega_{\delta}^{1}\left(\hat{M}^{-}, i \mathbb{R}\right)$ (we can assume we are working in a fixed based gauge). It is easy to verify that the section $s(\psi, \nu)=\not_{A_{0}+\nu} \psi$ is transverse to the zero set and therefore defines a parameterised space of harmonic spinors which maps to the parameter space $\Omega_{\delta}^{1}\left(\hat{M}^{-}, i \mathbb{R}\right)$ with a Fredholm map whose index coincides with the index of the Dirac operator. This implies that for generic $\nu$ the kernel of $\not_{A_{0}+\nu}$ has dimension zero. Now, as $\not_{A_{0}+\nu}$ is $\mathbb{C}$-linear, this kernel must have complex dimension greater than or equal to one or it vanishes. This proves the Lemma.

The Lemma tells us that, after suitable perturbation, we can deal with a smooth moduli space of reducible solutions, we can proceed as in the case of $M_{1}$ and we can again identify, for moduli spaces perturbed according to the hypothesis of the Lemma, $\mathcal{M}^{o}\left(P_{M_{2}}\right)=\mathcal{M}^{o}\left(P_{\hat{M}^{+}}\right)$. The moduli spaces on $M_{i}$ are therefore identified, and so are SW invariants. This completes the proof of the Proposition.

An equivalent proof of this result can be found by observing, as in [1, that we can consider $M_{2}=M_{1} \# \Sigma$, for $\Sigma$ a homotopy sphere (obtained by gluing $D_{\Gamma}^{4}$ with a standard disk along its boundary $S^{3}$ ), and then apply the previous ideas to obtain a connected sum formula for Seiberg-Witten invariants.

Now we pass to discuss the case of manifolds diffeomorphic outside a wedge of circles. We will prove:

Proposition 2.3. Let $M_{1}$ and $M_{2}$ be two manifolds with $b_{2}^{+}>1$ diffeomorphic in the complement of a wedge of circles. Then for any spin ${ }^{c}$ structure the $S W$ invariants of $M_{1}$ and $M_{2}$ coincide.

Proof. We assume now the existence of a diffeomorphism

$$
\phi: M_{1} \backslash \bigvee_{k} S^{1} \longrightarrow M_{2} \backslash \bigvee_{k} S^{1}
$$

In this case we consider, in a neighbourhood $U_{1} \subset M_{1}$ of the wedge, a standard $\#_{k} S^{1} \times S^{2}$ which bounds the boundary connected sum $\natural_{k} S^{1} \times D^{3}$, a tubular neighbourhood of the wedge; we can now decompose the two manifolds as

$$
M_{1}=M^{+} \cup_{\#_{k} S^{1} \times S^{2}}\left(\natural_{k} S^{1} \times D^{3}\right), \quad M_{2}=\phi\left(M^{+}\right) \cup_{\phi\left(\#_{k} S^{1} \times S^{2}\right)}\left(\natural_{k} S^{1} \times D^{3}\right)_{\Gamma} .
$$


We have, again, $\phi\left(M^{+}\right) \cong M^{+}$and as before finite energy moduli spaces on the associated cylindrical end manifolds coincide. We claim that the manifold $M_{2}^{-}$, as the notation of equation (2.7) suggests, has the same homotopy type of $\left(\natural_{k} S^{1} \times D^{3}\right)$.

Lemma 2.4. The manifold $M_{2}^{-}$is homotopy equivalent to $\left(\natural_{k} S^{1} \times D^{3}\right)$.

Proof. The proof consists in showing that $M_{2}^{-}$is an Eilenberg-Mac Lane space; for simplicity, we give the proof only in the $k=1$ case. We start by noting that, as removing cycles of codimension 3 does not change the first homotopy group, we have $\pi_{1}\left(M_{2}^{-}\right)=\mathbb{Z}$. Let us consider the universal covering $\tilde{M}_{2}^{-}$. We can construct it in the following (standard) way. Consider the projection $\pi: \partial M_{2}^{-} \cong$ $S^{1} \times S^{2} \rightarrow S^{1}$; the obstruction to extend this map to $M_{2}^{-}$lies in $H^{2}\left(M_{2}^{-}, \partial M_{2}^{-} ; \mathbb{Z}\right)=$ $H^{2}\left(M_{2}^{-}, M_{2}^{-} \backslash S^{1} ; \mathbb{Z}\right)$ where the latter equality follows from the diffeomorphism of pairs $\left(S^{1} \times D^{3} \backslash S^{1}, S^{1} \times S^{2}\right)=\left(M_{2}^{-} \backslash S^{1}, \partial M_{2}^{-}\right)$and the fact that the inclusion in the first pair is an homotopy equivalence. Using Alexander duality, we see that the obstruction space vanishes, so that $\pi$ extends to a map from $M_{2}^{-}$which induces an isomorphism of the fundamental groups. We take the inverse image $\Sigma$ of a regular value of $\pi$ and we cut open $M_{2}^{-}$along this submanifold, obtaining a four manifold with boundary diffeomorphic to $S^{2} \times[0,1] \cup \Sigma_{+} \cup \Sigma_{-}$; gluing an infinite chain of these manifolds along $\Sigma_{ \pm}$we obtain the universal covering, which is a simply connected manifold, with a boundary diffeomorphic to $S^{2} \times \mathbb{R}$ and diffeomorphic outside a curve $L$ to the cylinder $\left(D^{3} \backslash 0\right) \times \mathbb{R}$. Concerning its second homology group, it sits in the exact sequence

$$
H_{3}\left(\tilde{M}_{2}^{-}, \partial \tilde{M}_{2}^{-}\right) \stackrel{\delta}{\rightarrow} H_{2}\left(\partial \tilde{M}_{2}^{-}\right) \rightarrow H_{2}\left(\tilde{M}_{2}^{-}\right) \rightarrow H_{2}\left(\tilde{M}_{2}^{-}, \partial \tilde{M}_{2}^{-}\right)
$$

(we consider integer coefficients). The boundary $\partial \tilde{M}_{2}^{-}$is a deformation retract of $\tilde{M}_{2}^{-} \backslash L$; we can therefore identify $H_{2}\left(\tilde{M}_{2}^{-}, \partial \tilde{M}_{2}^{-}\right)=H_{2}\left(\tilde{M}_{2}^{-}, \tilde{M}_{2}^{-} \backslash L\right)$ and the latter vanishes as we can always disjoin, up to homotopy, a two chain in $\tilde{M}_{2}^{-}$from $L$. We have $H_{2}\left(\partial \tilde{M}_{2}^{-}\right)=\mathbb{Z}$. We claim that $\delta$ is surjective; to prove this, note that we can represent the generator of $H_{2}\left(\partial \tilde{M}_{2}^{-}\right)$as inverse image of a value $r \in \mathbb{R}$ of the projection map $\tilde{\pi}: \partial \tilde{M}_{2}^{-} \rightarrow \mathbb{R}$. This map can be extended continuously to a map $\tilde{\Pi}$ over the whole $\tilde{M}_{2}^{-} ;$therefore we can write $\tilde{\pi}^{-1}(r)=\delta \tilde{\Pi}^{-1}(r)$. Exactness of the sequence in equation (2.8) implies the vanishing of $H_{2}\left(\tilde{M}_{2}^{-}\right)$. Concerning the third homology group, we have, by retraction, $H_{3}\left(\tilde{M}_{2}^{-}\right)=H_{3}\left(i n t \tilde{M}_{2}^{-}\right)$and the latter homology group coincides with the compact support cohomology group $H_{c}^{1}\left(i n t \tilde{M}_{2}^{-}\right)$. This latter is a free abelian group whose rank equals the number of ends minus 1 . It is therefore trivial. Higher homology groups of $\tilde{M}_{2}^{-}$vanish by obvious dimension reasons. We deduce that $\tilde{M}_{2}^{-}$is contractible, and $M_{2}^{-}$has the homotopy type claimed in the statement.

For what concerns the homotopy and homeomorphism type of the manifolds involved in Proposition 2.3, it follows from our discussion that these manifolds are homotopy equivalent; if their fundamental group satisfies the hypothesis of Freedman Theorem, we deduce that they are homeomorphic too. When these hypotheses are not satisfied, we cannot decide whether $M_{1}$ and $M_{2}$ are homeomorphic or not.

We can now study the relation of the moduli spaces. Once again to prove the Proposition we are lead to consider the gluing theory along the manifold of positive scalar curvature $\#_{k} S^{1} \times S^{2}$; the result of Lemma 2.4 implies that $b_{2}\left(M_{2}^{-}\right)=0$ (so that reducibles can not be removed away) and that the reducible solutions on 
$\hat{M}_{2}^{-}$are identified, as a set, with $\chi(N)$. Also in this case there is a correspondence between $\operatorname{spin}^{c}$ structures on $M_{i}$ and $\operatorname{spin}^{c}$ structures on $M^{+}$which restrict to the trivial one on $N$.

Similarly to the case we discussed in the proof of Proposition [2.1, for what concerns $M_{1}$ the identification of $\mathcal{M}^{o}\left(P_{M_{1}}\right)$ with $\mathcal{M}^{o}\left(P_{\hat{M}^{+}}\right)$follows immediately from the fact that reducible solutions on the trivial $\operatorname{spin}^{c}$ structure on $\hat{M}_{1}^{-}$compose a nondegenerate moduli space $\chi\left(\hat{M}_{1}^{-}\right)=\chi(N)$ : the index of Dirac operator, coupled to a connection $A \in \chi\left(\hat{M}_{1}^{-}\right)$, is again zero and the kernel vanishes by curvature reasons. Equation (1.5) identifies the Zariski tangent space to $\chi\left(\hat{M}_{1}^{-}\right)$with $H^{1}(N, i \mathbb{R})$ which is actually its tangent space as a manifold. It is therefore nondegenerate and unobstructed. The fibre product of $\mathcal{M}^{o}\left(P_{\hat{M}^{+}}\right)$with $\chi\left(\hat{M}_{1}^{-}\right)$is then identified, as moduli space, with $\mathcal{M}^{o}\left(P_{\hat{M}^{+}}\right)$.

As in the case of Proposition 2.1 we have to do one more step before applying the gluing formula for $M_{2}$ as we cannot rule the vanishing of the kernel of the Dirac operator coupled with a connection in $\chi\left(\hat{M}_{2}^{-}\right)$, the moduli space of reducible solutions for the product $\operatorname{spin}^{c}$ structure on $\hat{M}_{2}^{-}$. In fact to solve this problem we need a result which generalises Lemma 2.2; we observe first of all that for a perturbation $\eta=d^{+} \nu$ as in equation (2.5) the set of reducible solutions of $d^{+} \nu$ SW equations is an "affine" $\chi\left(\hat{M}_{2}^{-}\right)$, i.e. solutions have the form $(A+\nu, 0)$ for $A \in \chi\left(\hat{M}_{2}^{-}\right)$; we will denote such a set as $\chi_{\nu}\left(\hat{M}_{2}^{-}\right)$. Information on this space is provided by the following:

Lemma 2.5. Let $M^{-}$be a manifold which has the homotopy type of $\sharp_{k} S^{1} \times D^{3}$. For a second category subset of perturbations $\eta=d^{+} \nu, \nu \in \Omega_{\delta}^{1}\left(\hat{M}^{-}, i \mathbb{R}\right)$ the set of solutions $(A, \psi)$ of the equation

$$
s_{\nu}(A, \psi)=\not_{A+\nu} \psi=0
$$

with nontrivial spinor is a smooth submanifold of $\chi\left(M^{-}\right) \times \Gamma_{\delta}^{*}\left(S^{+}\left(P_{\hat{M}^{-}}\right)\right)$of dimension $b_{1}\left(M^{-}\right)$; the natural projection map

$$
\pi: s_{\nu}^{-1}(0) \longrightarrow \chi\left(M^{-}\right)
$$

can be assumed to be transverse to any finite set of submanifolds and its fibre has real dimension at least 2 .

Proof. As we did in Lemma 2.2 in the case of a single flat connection, we have to study the zero set of the section $s(A, \psi, \nu)=\not \not_{A+\nu} \psi$ of the $\Gamma_{\delta}\left(S^{-}\left(P_{\hat{M}^{-}}\right)\right)$-bundle over $\chi\left(\hat{M}^{-}\right) \times \Gamma_{\delta}^{*}\left(S^{+}\left(P_{\hat{M}^{-}}\right)\right) \times \Omega_{\delta}^{1}\left(\hat{M}^{-}, i \mathbb{R}\right)$. On any fibre $\pi^{-1}(B)$, zero is a regular value, so that $s^{-1}(0)$ is a smooth submanifold, $\pi: s^{-1}(0) \rightarrow \chi\left(\hat{M}^{-}\right)$(that we can naturally identify with the limit map) is a submersion and has a Fredholm map to the parameter space of real index $2 i n d_{\mathbb{C}} \not_{A}+b_{1}\left(M^{-}\right)=b_{1}\left(M^{-}\right)$; for a generic choice of $\nu, s_{\nu}^{-1}(0)$ is therefore a $b_{1}\left(M^{-}\right)$-dimensional smooth manifold. Now, as observed above, the $\mathbb{C}$-linearity of Dirac equation ensures that, in correspondence of any connection $A$ in $\chi\left(\hat{M}^{-}\right)$such that $(A+\nu)$ has nonvanishing kernel, this kernel has real dimension at least two. This proves the statement on the dimension of the fibre.

It follows from the previous lemma that when we consider $\chi_{\nu}\left(\hat{M}_{2}^{-}\right)$, the points $A_{\nu}$ which are non-transverse (i.e. such that ker $\not_{A_{\nu}} \neq 0$ ) have the form $A_{\nu}=A+\nu$ with $A \in \pi\left(s_{\nu}^{-1}(0)\right)$. The set of these points (which is the natural analogue, in this context, of the theta divisor of a Jacobian torus) has zero measure in $\chi_{\nu}\left(\hat{M}_{2}^{-}\right)$and in 
particular the set of its limit values misses generically a finite set of points in $\chi(N)$.

This means that a generic flat connection on the boundary extends, on $\hat{M}_{2}^{-}$, to a regular reducible solution of equation (2.5). We have one more step to do to prove the equality of moduli spaces on $M_{1}$ and $M_{2}$, namely to prove that $\partial_{\infty}\left(\mathcal{M}\left(P_{\hat{M}^{+}}\right)\right)$ has dimension at most zero (and so it is at most a finite set of points). However, this follows from compactness and the dimension formula of equation (1.2), which shows that

$$
\operatorname{dim}\left(\mathcal{M}\left(P_{\hat{M}^{+}}\right)\right)=\operatorname{dim}\left(\mathcal{M}\left(P_{M_{2}}\right)\right)-2 i n d_{\mathbb{C}} \not_{A}=0
$$

as we work with zero dimensional moduli space because of simple type hypothesis. This result tells that we do not have to care about the eventual presence of nonregular reducible connections on $\hat{M}_{2}^{-}$, as $\partial_{\infty}\left(\mathcal{M}\left(P_{\hat{M}^{+}}\right)\right)$will avoid generically the flat connections, in $\chi(N)$, which extend to non-regular reducible connections. The gluing therefore is the same as we have described for the standard $\#_{\partial, k} S^{1} \times D^{3}$ and we have again $\mathcal{M}^{o}\left(P_{M_{2}}\right)=\mathcal{M}^{o}\left(P_{\hat{M}^{+}}\right)$. This concludes the proof of the Proposition.

\section{REFERENCES}

1. S.Demichelis, On Manifolds Diffeomorphic on the Complement of a Point, Rend.Mat.Acc. Lincei s.9,v.2 (1991), pp. 229-233. MR 92k:57060

2. S.Donaldson, P.Kronheimer, The Geometry of Four Manifolds, Oxford Math.Monographs, 1990. MR 92a:57036

3. S.Donaldson, D.Sullivan, Quasiconformal Four Manifolds, Acta Math.163 (1989), pp. 181-252. MR 91d:57012

4. R.Fintushel, R.Stern, 2-Torsion Instanton Invariants, J.Am.Math.Soc. 6 (1993), pp. 299-339. MR 93f:57038

5. R.Fintushel, R.Stern, Immersed Spheres in 4-Manifolds and the Immersed Thom Conjecture, Turk.J.Math. 19 (1995), pp. 145-157. MR 96j:57036

6. P.Kronheimer, T.Mrowka, The Genus of Embedded Surfaces in the Projective Plane, Math.Res.Lett. 1 (1994), pp. 797-808. MR 96a:57073

7. J.Morgan, T.Mrowka, D.Ruberman, The $\mathcal{L}^{2}$-Moduli Space and a Vanishing Theorem for Donaldson Polynomial Invariants, Series in Geometry and Topology, vol. 2, International Press Inc., 1994. MR 95h:57039

8. J.Morgan, Z.Szabó, C.Taubes, A Product Formula for Seiberg-Witten Invariants and the Generalized Thom Conjecture, J.Diff.Geom. 44 (1996), pp. 706-788. MR 97m:57052

9. C.Taubes, Self-dual Yang-Mills Connections on non Self-dual 4-Manifolds, J.Diff.Geom. 17 (1982), pp. 139-170. MR 83i:53055

10. S.Vidussi, Seiberg-Witten Theory for Four Manifolds Decomposed Along a Three Manifold of Positive Scalar Curvature, Prépublication École Polytechnique 99-5 (1999).

Centre de Mathématiques, UMR 7640 du CNRS, École Polytechnique, 91128 Palaiseau Cedex, France

Current address: Department of Mathematics, University of California, Irvine, California 92697

E-mail address: svidussi@math.uci.edu 